\title{
3D Human Models from 1D, 2D \& 3D Inputs: Reliability and Compatibility of Body Measurements
}

\author{
Alfredo BALLESTER*, Ana PIÉROLA, Eduardo PARRILLA, Jordi URIEL, \\ Ana V. RUESCAS, Cristina PÉREZ, Juan V. DURÁ, Sandra ALEMANY \\ Instituto de Biomecánica de Valencia (IBV), Universitat Politècnica de València, Valencia, Spain
}

DOI: $10.15221 / 18.132 \quad$ http://dx.doi.org/10.15221/18.132
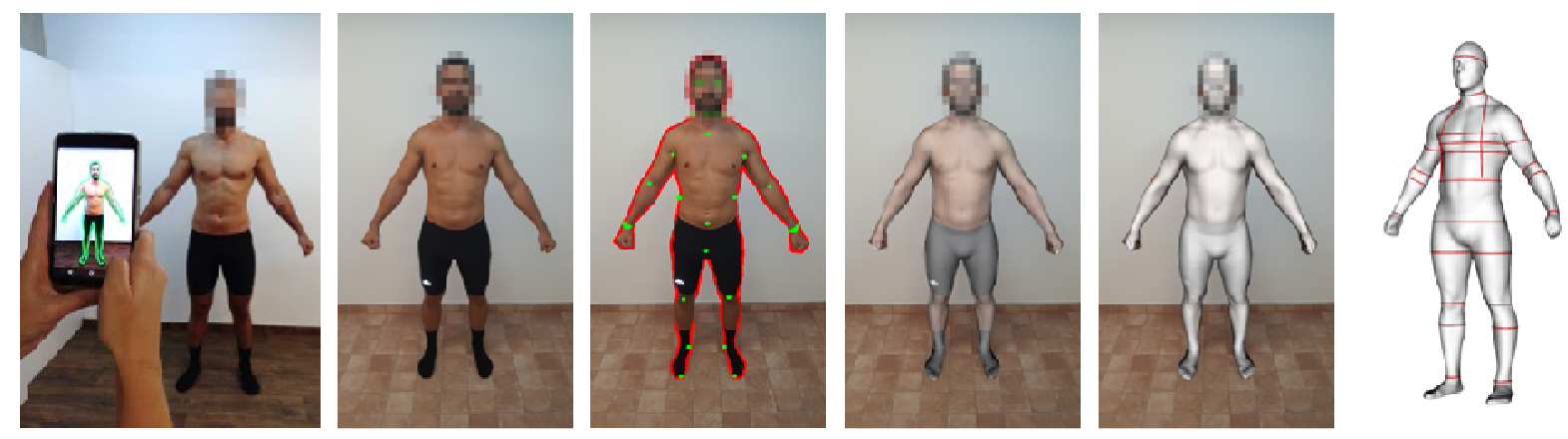

Figure 1. Data-driven 3D reconstruction of a male body from images taken with a smartphone app (2D3D)

\begin{abstract}
This paper presents partial results of a larger validation study of different Data-driven 3D Reconstruction (D3DR) technologies developed by IBV to create watertight 3D human models from measurements (1D3D), 2D images (2D3D) or raw scans (3D3D). This study quantifies the reliability (Standard Error of Measurement, SEM; Mean Absolute Deviation, MAD; Intra-class Correlation Coefficient, ICC; and Coefficient of Variation, CV) of body measurements taken on human subjects. Our results are also compared to similar studies found in literature assessing the reliability of digital and traditional anthropometry. Moreover, we assess the compatibility (bias and Mean Absolute Error, MAE) of measurements between D3DR technologies. The results show that 2D3D can provide visually accurate body shapes and, for the measurements assessed, 2D3D is as reliable as high resolution 3D scanners. It is also more accurate than manual measurements taken by untrained users. Due to accessibility, cost and portability (e.g. 2D3D built in a smartphone app) they could be more suitable than other methods at locations where body scanners are not available such as homes, medical or physical therapy offices, and small retail stores and gyms.
\end{abstract}

Keywords: Digital anthropometry, digital human models, body scanner, low-cost, data-driven, 3D reconstruction, PCA, measurements, accuracy, reliability, compatibility, SEM, ICC, MAD, MAE, CV.

\section{Introduction}

Human body shape and dimensions are useful for ergonomic product design [1]-[3]. Body dimensions are also used as biomarkers for the assessment of health conditions such as obesity and other cardio-metabolic risks [4]-[7]. Both accuracy and reliability are indispensable for these purposes.

Measuring human subjects is challenging because our bodies are soft and articulated. Even standardised body measuring procedures [8]-[10] contain an inherent variability due to respiration phase and slight differences in pose and muscle contraction.

Historically, the standard method for measuring bodies for product design and health assessment has been traditional anthropometry (TA). This technique requires a trained measurer and specialised metrology instruments such as callipers for distances (heights, breadths, depths and widths) and measuring tape for girths and lengths over the body [11]. Its accuracy and reliability depend on the skills of the measurer. Usually there are systematic biases between measurers which result in larger inter-observer errors [12]-[15]. In the context of size selection of apparel and orthotics products in catalogue orders and online purchases, the most common method is to request the buyer to self-report a few measurements taken following instructions that the brand or retailer provides to the buyer.

\footnotetext{
* alfredo.ballester@ibv.org; +34 610562 532; http://anthropometry.ibv.org
} 
Measurements reported by untrained buyers are more unreliable and inaccurate than those obtained by trained experts [16].

The release of the first commercial 3D body scanners in the late nineties revolutionised the methods for body measuring [17], [18]. They also made possible the use of the actual body shapes, and not just measurements, in digital product design [19]-[23]. Body scanners can acquire the body surface of minimally dressed human bodies and measure them digitally. The main sources of error of measurements obtained by body scanning that affect reliability are: resolution and 3D accuracy of the scanner, body sway during scanning, slight pose changes between repeated scans, the measurement extraction software, and, for body scanning software that requires interactive inspection and landmark editing, also the skills of the inspector [24]. Digital measuring of human bodies can be as reliable as trained experts using TA [25], [26]. Body scanners are also more efficient, since they can take an unlimited number of measurements in the same time that an expert takes 5-10 measurements [25]. However, the compatibility between measurements obtained by different body scanners and measuring software is still an open issue being addressed by different standardization bodies such as ISO and IEEE [27]-[29]. Despite their precision and efficiency, body scanners are too bulky and expensive to be used at certain environments such as small retail stores, medical offices and homes.

Thanks to the large databases created using 3D body scanners [30]-[32], lower-cost technologies based on data-driven 3D reconstructions (D3DR) have been developed to estimate human body models from a few measurements (1D3D) or from 2D images (2D3D) [33]-[37]. D3DR solutions have much lower hardware requirements than actual 3D scanners. These solutions are suitable for running in small portable devices such as phones or tablets, making it possible to have a body scanner in your pocket. D3DR has also been applied to enhance raw data (3D3D), namely by robustly completing missing data and by correcting noise and artefacts of 3D scans from lower-cost scanners [38]-[41].

In this study, we quantified the reliability of body measurements of 3D models obtained using D3DR technologies developed by IBV, i.e. from two images taken with a smartphone app (2D3D) and from raw scans (3D3D). Secondly, we assessed the reliability of 2D3D by comparing it with 3D3D and with well-established methods reported in literature, in particular with 3D body scanning (3DBS) and with traditional anthropometry (TA). Thirdly, we assessed the compatibility of measurements between D3DR technologies, i.e. 1D3D (using three different sets of user-reported measurements as input), 2D3D and 3D3D. Finally, we discuss the potential of the D3DR technologies assessed and their suitability for different product design and health applications.

\section{Materials and methods}

\subsection{Design of experiment}

A total of 77 subjects participated in the study (39 females and 38 males). Subjects were selected to cover a wide range of body height and weight (Table 1). This number of subjects enables us to analyse reliability in our sample both as a whole and by gender, according to optimal experiment design methods [42] for a test reliability hypothesis at $5 \%$ significance level with $80 \%$ power, a minimal acceptable ICC of 0.8 and an expected ICC of 0.95 , and two repetitions (min. no. of subjects $=13$ ).

Table 1. Descriptive statistics Mean $\pm S D$ (range) of the sample of subjects by gender

\begin{tabular}{lccc}
\hline & Female $\mathrm{N}=39$ & Male $\mathrm{N}=38$ & Total $\mathrm{N}=77$ \\
\hline Age (years) & $33 \pm 10(19-58)$ & $35 \pm 8(20-52)$ & $34 \pm 9(19-58)$ \\
Weight $(\mathrm{Kg})$ & $61.2 \pm 9.1(44.3-95.8)$ & $78.9 \pm 15.2(52.8-136)$ & $69.9 \pm 15.3(44.3-136)$ \\
Height $(\mathrm{cm})$ & $165 \pm 6(149-175)$ & $175 \pm 8(160-189)$ & $170 \pm 9(149-189)$ \\
\hline
\end{tabular}

All participants were measured with our 2D3D app running on a Motorola Nexus 6 and with Vitus XXL body scanner from Human Solutions $\mathrm{GmbH}$. Each subject was scanned and photographed twice with repositioning, getting in and out of the scanning/photographing space at each repetition. Subjects wore tight-fitting clothes when measured with both technologies and used a swimming cap in the scanner. Subject's body mass and height were also measured using a SECA weighing scale and a stadiometer.

A subset of 37 participants randomly selected (20 females and 17 males) were also requested to report their body measurements taken at home prior to the scanning session following typical instructions found in size guides from online stores. The self-reported measurements and parameters were: age, weight, height, bust/chest girth, waist girth, hip girth, crotch height, underbust girth (only females) and mid neck girth (only males). 


\subsection{Data processing using Data-driven 3D Reconstruction (D3DR) methods}

Gathered data (reported measurements, images and raw scans) was used to create watertight 3D models using three different D3DR methods developed by IBV: 1D3D, 2D3D and 3D3D (Figure 2). Table 2 summarises the inputs used for each method.

Table 2. Summary of D3DR inputs used

\begin{tabular}{c|l}
\hline Method & Input data \\
\hline 1D3D(3) & Age, Height, Weight \\
1D3D(6) & $\begin{array}{l}\text { Age, Height, Weight, Chest } \\
\text { girth, Waist girth, Hip girth }\end{array}$ \\
1D3D(7) & $\begin{array}{l}\text { Age, Height, Weight, Chest } \\
\text { girth, Waist girth, Hip girth, } \\
\text { Crotch height }\end{array}$ \\
2D3D & $\begin{array}{l}\text { Age, Height, Weight, front } \\
\text { image, side image }\end{array}$ \\
3D3D & \begin{tabular}{l} 
Raw 3D scan \\
\hline
\end{tabular}
\end{tabular}

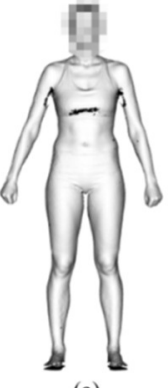

(a)

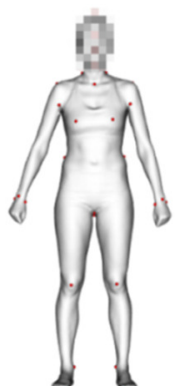

(b)

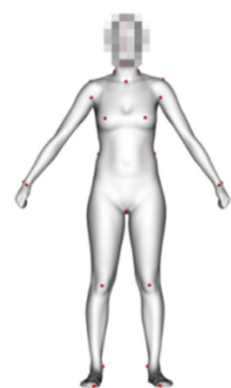

(c)

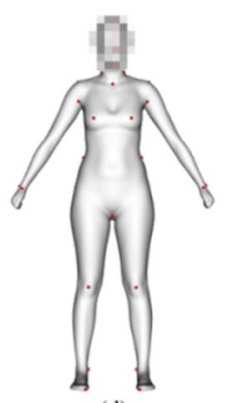

(d)

Figure 2. 3D body reconstructions of the same female body using different technologies. (a) raw scan, (b) $3 D 3 D$ reconstruction, (c) $2 D 3 D$ reconstruction and $(d) 1 D 3 D$ reconstruction

All our methods rely on parametrised spaces of human body shape using Principal Component Analyses (PCA) learnt from more than 9.000 body scans registered using template-fitting methods. Our implementations are improvements on previously reported methods or alternatives to methods reported in literature:

- 1D3D - Creation of a watertight 3D model from a set of parameters such as age or measurements. This technology can be configured to use different sets of inputs. In this study, we created three 1D3D models for each user using different sets of measurements (Table 2). Our implementation consists of learning a linear mapping between body measurements and the PCA weights. The user-reported measurements were used as inputs to 1D3D reconstruction [19].

- 2D3D - Creation of a watertight 3D model from two images taken with a smartphone app (figure 1, page 1). Our implementation consists of an Android app client that helps the user to take the photographs and a cloud processing service via API. It is an improvement of previously reported methods [43] which uses machine learning and artificial Intelligence (AI) at segmentation and landmarks detection steps [44], [45] and an improved weighting of the body parts between front and side silhouettes in shape optimization steps. Our new method makes the process much more robust to the most common usability pitfalls, in particular: to hairstyles that increase apparent height (Figure 3), to not correctly matching the guiding outline when taking the photographs (Figure 4), to bad image contrast/lighting (Figure 5), and to not finding a location free of objects (Figure 6), to slightly incorrect arms/legs' pose (Figure 7).

- 3D3D - Creation of a watertight 3D model from a raw 3D scan (Figure 8). Our implementation uses a shape and pose model alternative to the methods described by other authors [38], [40]. This method is more robust to scanner artefacts than previously reported methods [19] making it possible to automatically process raw body scans of different quality (incl. very noisy and highly artefacted ones) in A-Pose without landmarks [39].

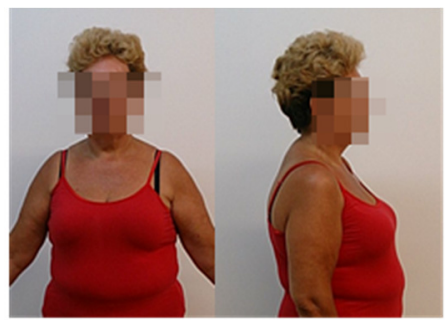

Input image

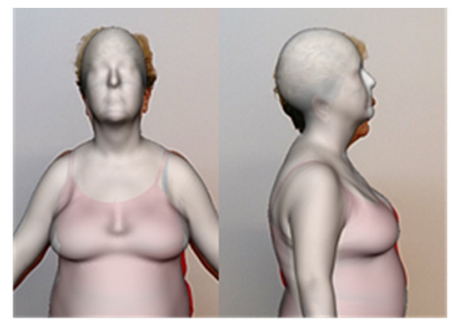

old method [43]

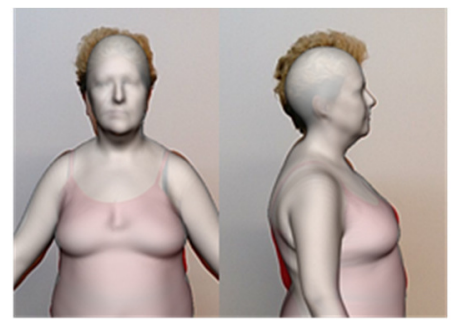

new method

Figure 3. 2D3D reconstruction robust to hairstyles that increase apparent height 


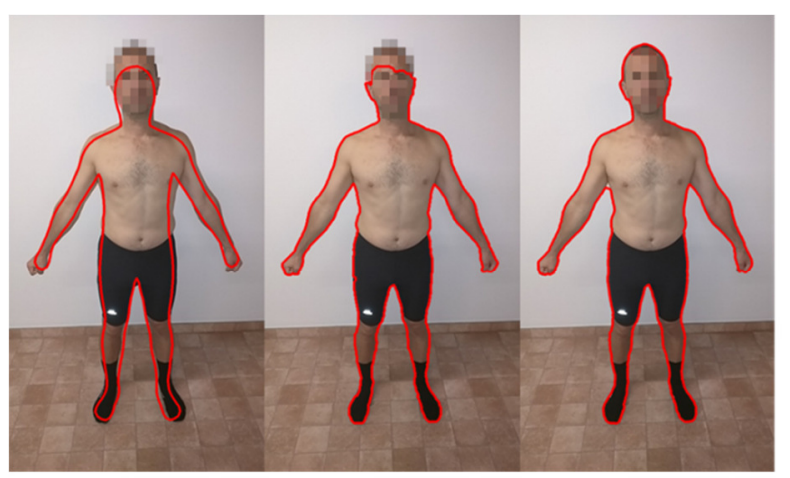

Poor guide outline fit old method [43]

new method

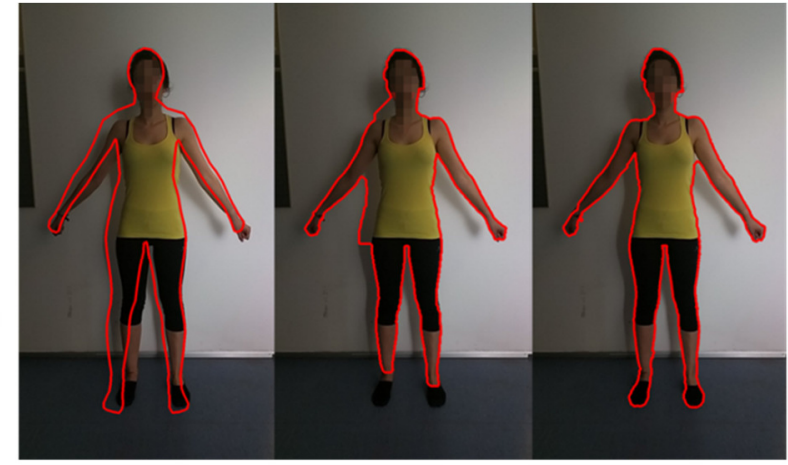

Poor guide outline fit old method [43]

new method

Figure 4. Robust segmentation to poor guiding outline fit when taking photographs

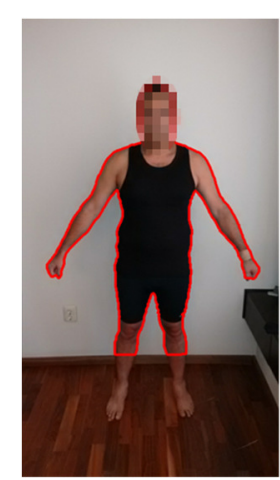

old method [43]

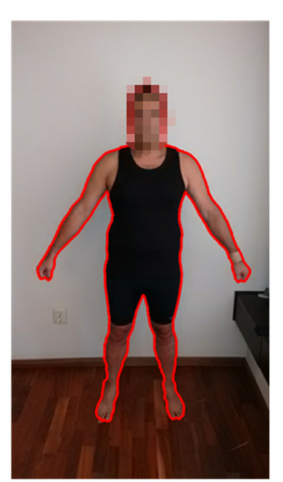

new method

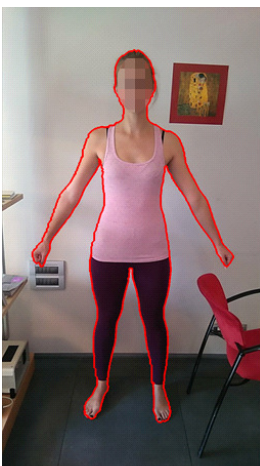

new method

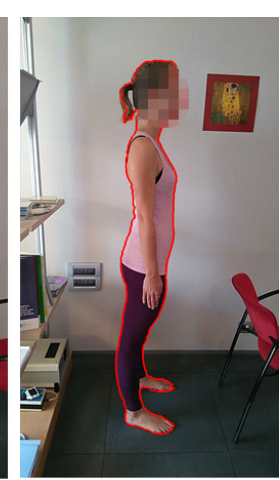

new method

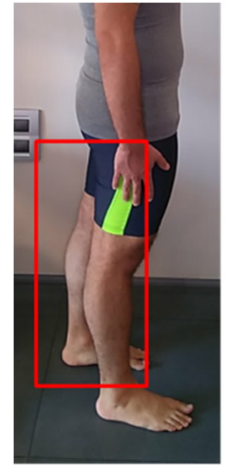

Back leg visible

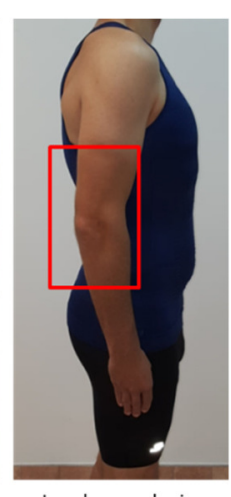

Lumbar occlusion
Figure 6. Robust segmentation to scenes with objects
Figure 7. 2D3D reconstruction robust to bad arm/leg pose to poor colour contrast

High resolution

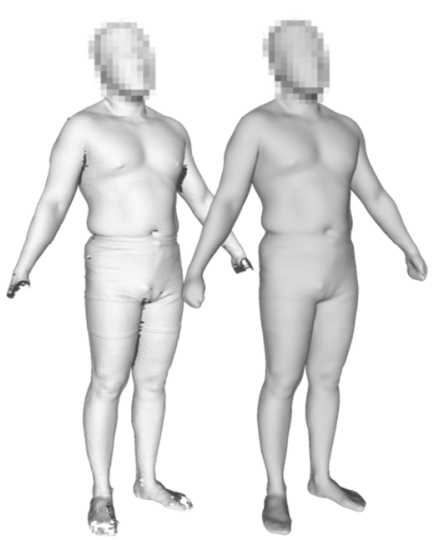

Low resolution

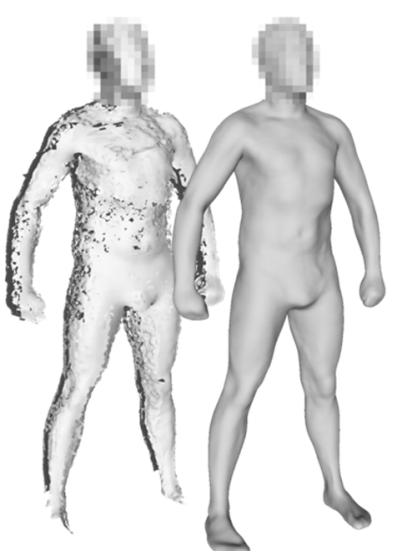

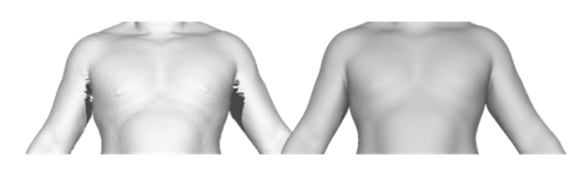

Data-driven anatomical slit modelling, denoising \& geometry completion

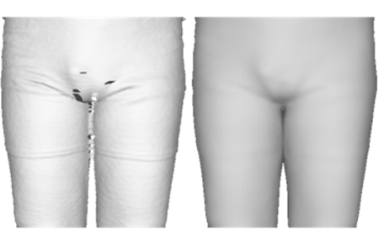

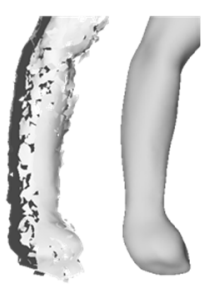

Figure 8. Examples of 3D3D reconstruction of a high resolution scan and a noisy scan

All 3D models were measured using our digital measuring tape, which was developed to be compatible with most of the ISO 8995-1:2017 definitions [19]. Our digital measuring tape does not require visual inspection or interactive correction of the measurements and landmarks. 100 measurements were extracted, from which 17 were selected to be included in this paper due to its relevance.

\subsection{Analytic procedures}

Reliability: We estimated the Standard Error of Measurement (SEM) and Intra-class Correlation Coefficient (ICC) for each measurement and each technology (i.e. 2D3D and 3D3D) using the methods proposed by [46]. We also estimated the Mean Absolute Deviation (MAD) and the Coefficient of Variation (CV). 
Compatibility: We estimated the signed mean difference (Bias) and the Mean Absolute Error (MAE) for each measurement between pairs of methods. In this paper we report the compatibility between 3D3D and the other four methods, i.e. 2D3D, 1D3D(3), 1D3D(6), 1D3D(7), and self-reported measurements, using 3D3D as reference method.

Additionally, we analysed the methods and the errors reported by users during the manual measuring session.

\section{Results and discussion}

\subsection{Reliability}

SEM and MAD of the solutions assessed are presented in Table 3. We also compare our results to other reliability studies of measurements digitally extracted from raw body scans (3DBS) and measurements taken by experts with traditional anthropometric methods (TA).

Table 3. Mean Absolute Difference (MAD) and Standard Error of Measurement (SEM) in centimeters of D3DR solutions (2D3D, 3D3D) and of 3D Body scanners (3DBS) and Traditional Anthropometry (TA) reported in literature

\begin{tabular}{lcccc} 
& \multicolumn{2}{c}{ This study } & \multicolumn{2}{c}{ Other studies } \\
\cline { 2 - 5 } Measurement & 3D3D & 2D3D & 3DBS & TA \\
MAD (SEM) & MAD (SEM) & MAD (SEM) & MAD (SEM) \\
\hline Height & $0.1(0.3)$ & $-\mathrm{a}$ & $0.2-0.4(0.4-0.5)$ & $0.1-0.7(0.5)$ \\
Cervical height & $0.1(0.2)$ & $0.3(0.5)$ & $0.3-0.4(0.3-0.5)$ & $0.2-0.7(-)$ \\
Crotch height & $0.1(0.3)$ & $0.3(0.6)$ & $0.416(0.4-1)$ & $0.5-0.5(-)$ \\
\hline Mid neck girth & $0.1(0.3)$ & $0.3(0.5)$ & $0.5-0.5(0.7-1.3)$ & $0.3-0.4(-)$ \\
Shoulder width & $0.5(0.8)$ & $0.5(1)$ & $1.208(0.8-1.2)$ & $0.433(-)$ \\
Shoulder length & $0.1(0.2)$ & $0.2(0.3)$ & $0.8-0.8(-)$ & $0.2-0.2(-)$ \\
Shoulder breadth & $0.3(0.5)$ & $0.3(0.5)$ & $0.6-1.4(-)$ & $0.2-0.9(-)$ \\
\hline Bust/chest girth & $0.4(0.7)$ & $0.5(1)$ & $0.6-1.2(0.8-2.6)$ & $0.5-1.8(8.2)$ \\
Underbust girth & $0.4(0.7)$ & $0.5(1)$ & $1.444(1.2-2)$ & $0.592(-)$ \\
Waist girth & $0.4(0.7)$ & $0.6(1)$ & $0.5-0.9(0.7-3.3)$ & $0.5-1.6(1.3-6.5)$ \\
Hip girth & $0.2(0.4)$ & $0.5(0.8)$ & $0.2-0.5(0.4-2.6)$ & $0.4-1.4(6.8)$ \\
\hline Arm length & $0.2(0.4)$ & $0.5(0.9)$ & $0.5-1.2(0.7-0.8)$ & $0.3-0.8(-)$ \\
Upper arm girth & $0.1(0.2)$ & $0.3(0.5)$ & $0.75(0.4-0.9)$ & $0.3-0.6(-)$ \\
Wrist girth & $0.1(0.2)$ & $0.2(0.3)$ & $0.3-0.3(0.2-0.5)$ & $0.1-0.3(-)$ \\
\hline Max thigh girth & $0.1(0.3)$ & $0.3(0.6)$ & $0.47(0.2-1.4)$ & $0.3-0.9(-)$ \\
Knee girth & $0.1(0.3)$ & $0.2(0.3)$ & $0.3-0.3(0.2-0.9)$ & $0.26-0.33(-)$ \\
\hline Body Volume & $0.01(0.02)$ & $0.05(0.1)$ & $0.024(0.03-0.06)$ & - \\
a Not applicable because the system uses body height to scale the solution & {$[15],[26],[47]-[55]$} & \\
b Range of values for 3D Body Scanners (3DBS) from literature & {$[12]-[15],[24]-[26],[56]-[59]$} \\
c Range of values for Traditional Anthropometry (TA) from literature
\end{tabular}

The results show that the SEM of 3D3D modelling and measuring method is within $0.2-0.8 \mathrm{~cm}$ and that $M A D$ is within $0.1-0.5 \mathrm{~cm}$. One part of the error found in 3D3D results is due to technology (3D accuracy, resolution and measuring and modelling software) and another part is due to body sway during scanning and to slight pose changes between repeated scans [24], [60]. An experiment with a variety of rigid body shapes of real humans would be required in order to estimate the order of magnitude of each error source. ICC is within 0.983-0.999 and CV is below $2 \%$.

The SEM of 2D3D modelling and measuring method is within $0.3-1.0 \mathrm{~cm}$ and that MAD is within 0.2-0.6 $\mathrm{cm}$. The small error increases with respect to 3D3D are due to using much less input information (2D vs. 3D) gathered with less reliable technology (smartphone camera vs. body scanner sensors). ICC is within $0.938-0.997$ and $\mathrm{CV}$ is below $2 \%$.

SEM and MAD of measurements obtained from 3D raw body scans (3DBS) reported in bibliography by different authors (and using different hardware and software) range respectively from 0.2-3.3 cm and from 0.2-1.4 cm [15], [26], [47]-[55], [61]. SEM and MAD of measurements obtained by traditional anthropometry (TA) reported in bibliography range respectively from $0.1-1.8 \mathrm{~cm}$ and from $0.5-8.2 \mathrm{~cm}$ [12]-[15], [24]-[26], [56]-[59]. The reliability of the self-reported measurements by untrained users is expected to be at best in the order of magnitude of the worst values for experts (e.g. SEMs in the range of 5-8 $\mathrm{cm}$ for large girths). 
3D3D and 2D3D technologies provide SEM, MAD, ICC and CV values within the range of best results reported by other studies using 3DBS or TA. 3D3D exhibited even slightly better reliability for some measurements. ICCs of both technologies for all measurements are excellent ( $>0.9)$ [62]. MAD also lies within the allowable error established for garment construction and ergonomic design [13], [61].

\subsection{Compatibility}

Six examples of the reconstructed bodies with 3D3D and 2D3D technologies are provided in Figure 9. It can be observed that the body shapes of 2D3D reconstructed bodies are very similar those resulting from actual scanning (3D3D).
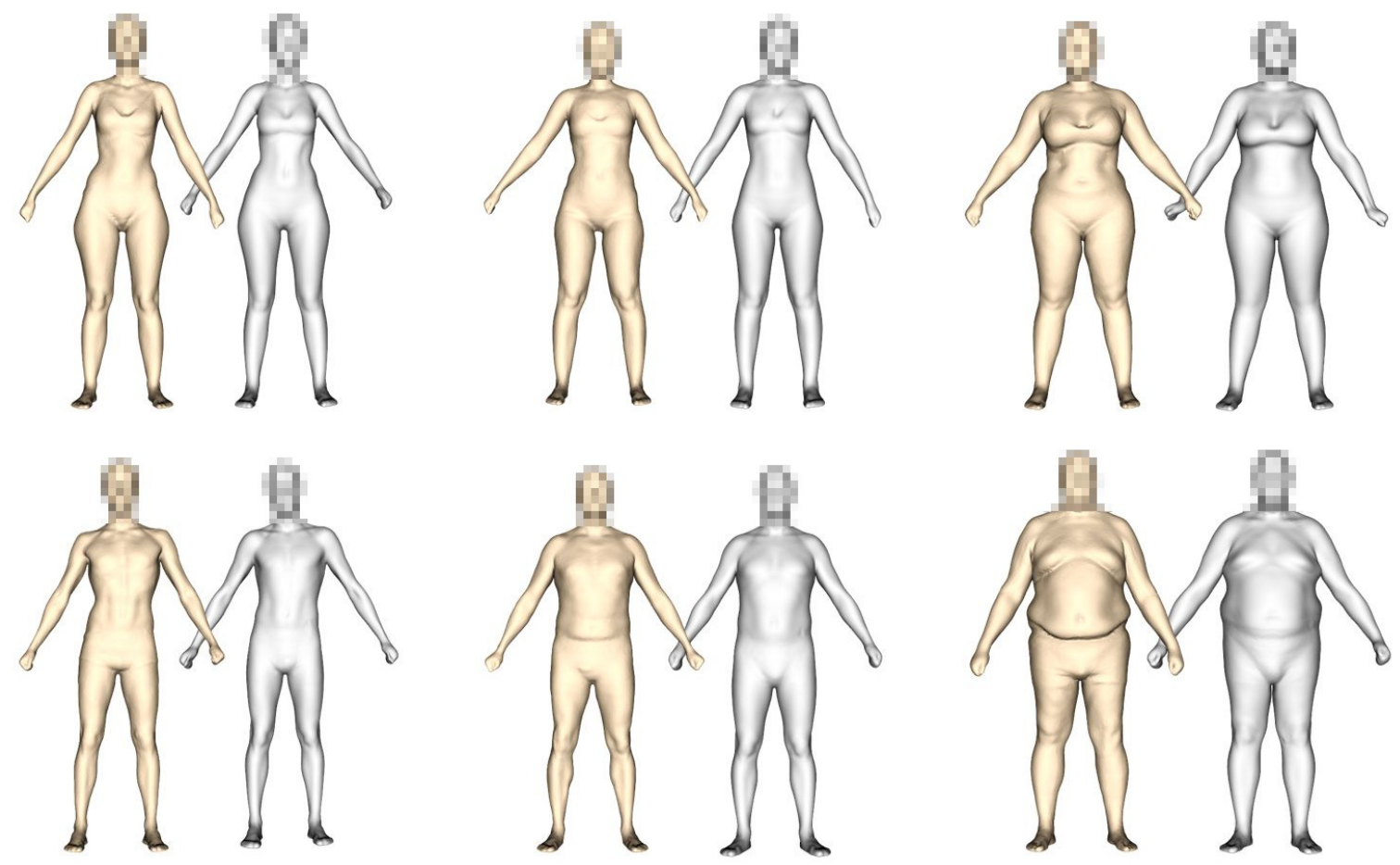

Figure 9. $3 D$ models of six subjects: 3D3D (golden, left) and 2D3D reconstructions (silver, right)

Bias and MAE between 3D3D and lower-cost alternatives (self-reported measurements, 2D3D and the 3 configurations 1D3D) are presented in Table 4. Regarding the self-reported measurements taken at home, 12 of the 37 subjects (32\%) required help from a second person to help them to take body girth measurements, and $17(46 \%)$ needed help to take crotch height measurement. Five of the 37 subjects $(13 \%)$ took the girth measurements incorrectly: one subject read the measuring tape on the wrong direction and the rest took half of the body contour instead of the full girths. Self-reported data from these five subjects was excluded from the analyses and not used as input for 1D3D reconstruction.

Compared to the body scans processed with 3D3D, when users reported their measurements, they overestimated their body height $(-1.4 \mathrm{~cm})$, mid neck girth $(-0.8 \mathrm{~cm})$ and crotch height $(-4.8 \mathrm{~cm})$ and underestimated their chest $(2.4 \mathrm{~cm})$, waist $(2 \mathrm{~cm})$ and hip $(4.1 \mathrm{~cm})$. These differences are largely beyond the bias threshold established by ISO 20865:2010 [63]. MAE of self-reported measurements is within 1.5-5.0 cm, which is largely beyond MAE thresholds established by ANSUR [13], except for body height which is closer to it.

Biases of 1D3D(3) are within $\pm 1.4 \mathrm{~cm}$ except for bust girth, which is underestimated by $4.2 \mathrm{~cm}$. Since biases of input measurements (crotch height, bust/chest and hips) are significant, biases of 1D3D(6) and 1D3D(7) are increased, except for bust/chest girth which improves slightly. MAE of the three configurations of 1D3D is similar, even worsening when measurement inputs are highly inaccurate like crotch height $(5 \mathrm{~cm})$ and hip girth $(4.6 \mathrm{~cm})$. Examples of the visual comparisons of the 1D3D reconstructions to 3D3D models are provided in Figure 10. In some of these examples, the addition of measurements just slightly improves the 1D3D reconstructions. Examples of severe errors in input measurements are illustrated in Figure 11 (error in chest girth) and Figure 12 (error in crotch height). In these two examples, the addition of measurements leads to more inaccurate 1D3D reconstructions. 
Table 4. Signed Mean Difference (Bias) and Mean Absolute Error (MAE) in centimeters between measurements obtained using 3D3D and lower-cost alternatives (self-reported, 2D3D and 1D3D)

\begin{tabular}{|c|c|c|c|c|c|c|}
\hline Measurement & $\begin{array}{r}\text { 3D3D - } \\
\text { 2D3D } \\
\text { Bias (MAE) }\end{array}$ & $\begin{array}{l}\text { 3D3D - } \\
\text { self-rep } \\
\text { Bias (MAE) }\end{array}$ & $\begin{array}{l}\text { 3D3D - } \\
\text { 1D3D(3) } \\
\text { Bias (MAE) }\end{array}$ & $\begin{array}{l}\text { 3D3D - } \\
\text { 1D3D(6) } \\
\text { Bias (MAE) }\end{array}$ & $\begin{array}{l}\text { 3D3D - } \\
\text { 1D3D(7) } \\
\text { Bias (MAE) }\end{array}$ & $\begin{array}{l}\text { Max. Allowable } \\
\text { Error [13], [63] } \\
\text { Bias (MAE) }\end{array}$ \\
\hline Height & $0.03(0.8)$ & $-1.4(1.6)$ & $-1.3(1.9)^{b}$ & $-1.4(1.9)^{b}$ & $-1.5(2)^{b}$ & $0.5(1.1)$ \\
\hline Cervical height & $0.2(1)$ & - & $-1.4(1.8)$ & $-1.3(1.7)$ & $-1.9(2.2)$ & $0.5(0.7)$ \\
\hline Crotch height & $-0.1(1.1)$ & $-4.8(5.0)$ & $-1.4(1.7)$ & $-1.3(1.6)$ & $-4.4(4.6)^{b}$ & $0.5(1.0)$ \\
\hline Mid neck girth & $-0.6(1.1)$ & $-0.8(1.5)$ & $-0.8(1.2)$ & $-0.7(1.1)$ & $-0.5(1)$ & $0.4(0.6)$ \\
\hline Shoulder width & $-1.8(2.2)$ & - & $0.4(1.9)$ & $0.4(1.9)$ & $0.6(1.9)$ & $0.4(-)$ \\
\hline Shoulder length & $0.1(0.4)$ & - & $0.3(0.4)$ & $0.2(0.4)$ & $0.4(0.5)$ & $0.5(0.3)$ \\
\hline Shoulder breadth & $0.5(1.0)$ & - & $0(1.1)$ & $-0.1(1.2)$ & $0.3(1.2)$ & $0.4(0.8)$ \\
\hline Bust/chest girth & $1.1(1.7)$ & $2.4(2.9)$ & $4.2(4.3)$ & $3.5(3.6)^{b}$ & $3.5(3.6)^{b}$ & $0.9(1.5)$ \\
\hline Underbust girth & $-0.7(1.4)$ & $0.4(2.7)$ & $1.6(1.9)$ & $2.4(2.6)$ & $2.3(2.5)$ & $0.9(1.6)$ \\
\hline Waist girth & $0.5(1.6)$ & $2(3)$ & $0.6(3.4)$ & $2(3.1)^{b}$ & $1.8(3)^{b}$ & $0.9(1.1)$ \\
\hline Hip girth & $-0.4(1.5)$ & $4.1(4.6)$ & $1.4(3.1)$ & $1.7(3.2)^{b}$ & $2.2(3.7)^{b}$ & $0.9(1.2)$ \\
\hline Arm length & $0.3(1.3)$ & - & $-1.1(1.6)$ & $-1(1.6)$ & $-2.1(2.3)$ & $0.5(-)$ \\
\hline Upper arm girth & $0.4(1.2)$ & - & $0.9(1.4)$ & $0.8(1.4)$ & $0.9(1.4)$ & $0.5(0.6)$ \\
\hline Wrist girth & $-0.6(0.8)$ & - & $-0.2(0.7)$ & $-0.2(0.7)$ & $-0.1(0.7)$ & $0.5(0.5)$ \\
\hline Max thigh girth & $-0.9(1.3)$ & - & $0.8(2.2)$ & $0.8(2.0)$ & $0.6(2.0)$ & $0.5(0.6)$ \\
\hline Knee girth & $-0.8(1.2)$ & - & $-0.2(1.4)$ & $-0.1(1.4)$ & $-0.2(1.4)$ & $0.5(0.4)$ \\
\hline Full body volume (I) & $-0.1(0.2)$ & - & $0.1(0.2)$ & $0.1(0.3)$ & $0.2(0.3)$ & - \\
\hline
\end{tabular}

${ }^{a}$ Results with the 32 subjects retained (5 subjects' discarded because they were unable to properly take measurements)
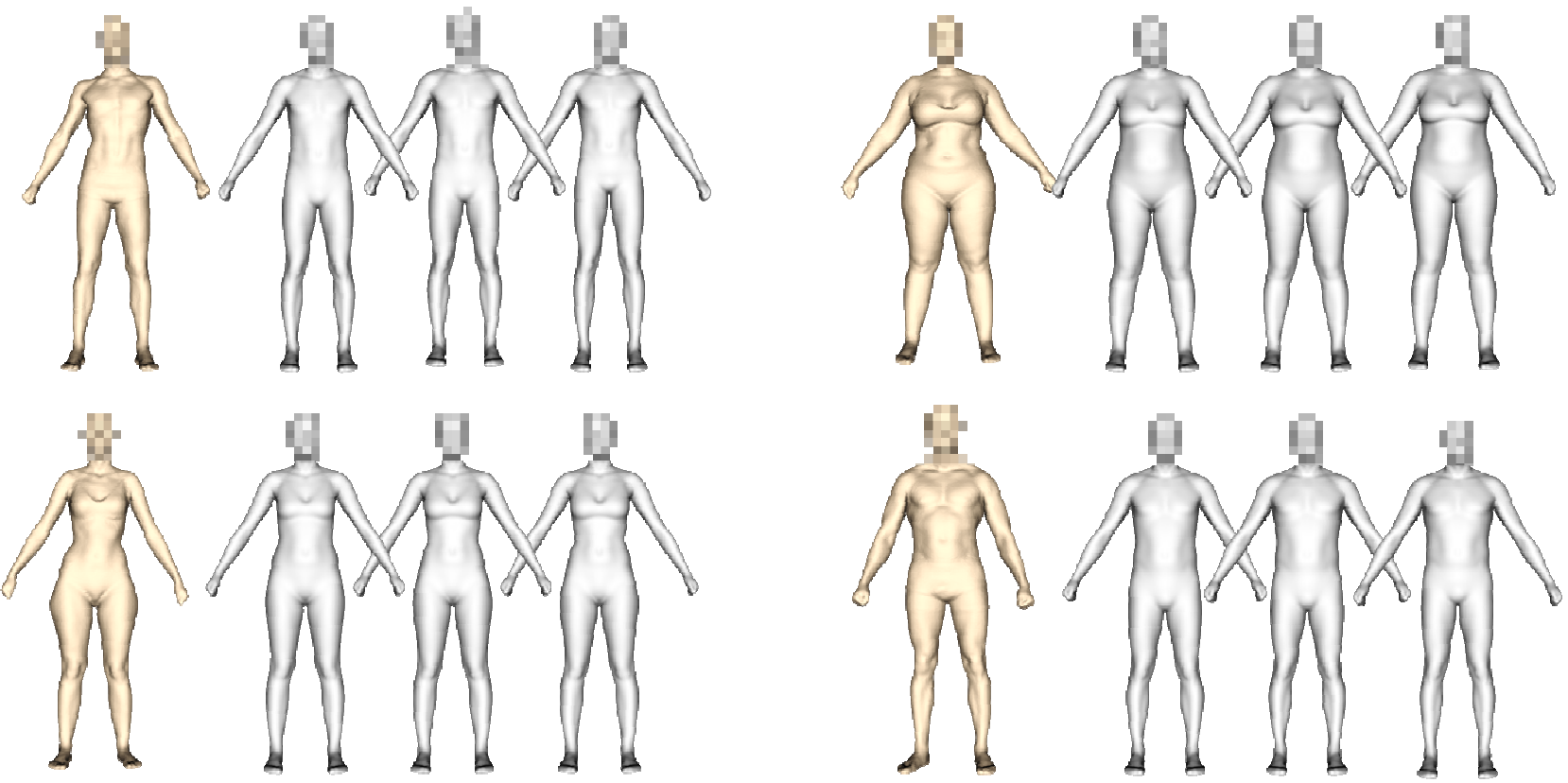

Figure 10. 3D models of four subjects: 3D3D (golden, left) and 1D3D with 3, 6 and 7 parameters (silver, right)

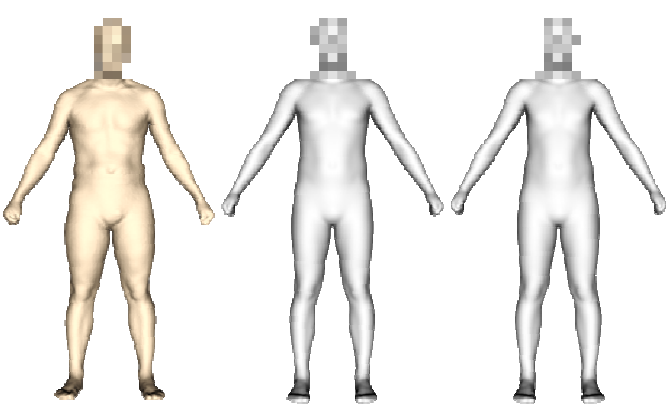

Figure 11. Example of 1D3D(3) that worsens in $1 D 3 D(6)$ by adding inaccurate chest girth

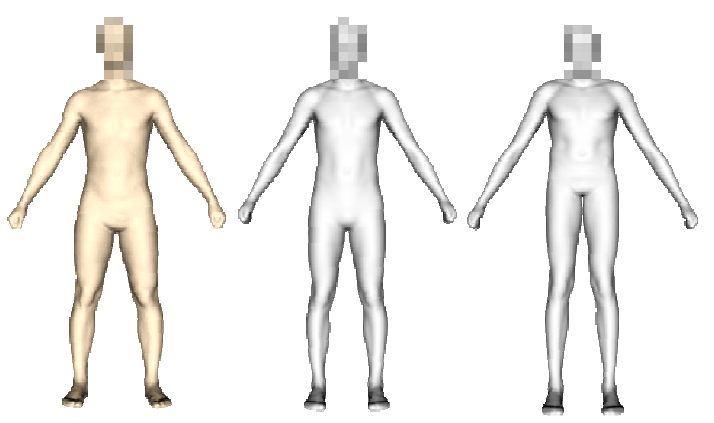

Figure 12. Example of 1D3D(3) reconstruction that worsens in $1 D 3 D(7)$ by adding inaccurate crotch height 
Biases of 2D3D with respect to 3D3D are within $\pm 1.1 \mathrm{~cm}$ except for Shoulder width $(-1.8 \mathrm{~cm})$. For most measurements, bias is within the threshold established by ISO 20685:2010 or close to it, except for thigh and knee girths. MAE of 2D3D is within $0.4-1.7 \mathrm{~cm}$, except for Shoulder width $(2.2 \mathrm{~cm})$. For most measurements, MAE is within the threshold established by ANSUR or close to it, except for upper arm, thigh and knee girths, which are below $1 \mathrm{~cm}$. MAE is lower than the six self-reported measurements and lower than all measurements resulting from the three configurations of 1D3D, except for Shoulder width. In particular, for critical measurements such as bust/chest, waist and hip girths, MAE is around $1.5 \mathrm{~cm}$ compared to $3-4 \mathrm{~cm}$ for the self-reported and 1D3D. The slightly larger biases and MAE of shoulder width will be further studied and improved in future versions.

\section{Conclusions and further work}

D3DR methods can provide benefits to the processing of raw scans (3D3D) and to reconstruct 3D shapes from sparse information such as images (2D3D) or parameters (1D3D). Single-surface watertight 3D models obtained with the three methods are high-resolution meshes ready to interface other CAD and simulation applications.

Our fully automatic and unsupervised 3D3D modelling and measuring methods proved to be as reliable as the best expert measurers, achieving a SEM below $0.7 \mathrm{~cm}$ for critical body girths at mid-neck, bust/chest, waist and hip, and below 0.4 at critical lengths and heights (i.e. cervical height, crotch height and arm length). A prototype of this technology has been implemented in a cloud server accessible via $\mathrm{API}$ and it is ready to be tested by different scanning vendors and 3D scanner users.

Our 2D3D reconstruction from two images can be implemented in a regular smartphone thus making it accessible to millions of people without the need of specific hardware or skills. 2D3D provides visually accurate 3D models and the reliability of measurements obtained from these models is comparable to body scanners (CV below $2 \%$, MAD below $0.5 \mathrm{~cm}$ and SEM below $1 \mathrm{~cm}$ ). Its accuracy for most measurements (MAE below $1.7 \mathrm{~cm}$ ) is much higher than self-reported measurements by users (MAE within 1.5-5.0 cm). All these features make it more suitable than self-reported measurements for any application or context where body scanning cannot be available, such as medical offices, homes and small retail stores. A prototype for Android OS has been developed and it is ready to be tested in real contexts. Moreover, we are exploring variants of the method to enable a single person to take the pictures with the front camera (selfie point of view from the floor level) and the 3D reconstruction of people dressed with street clothes (Figure 13).
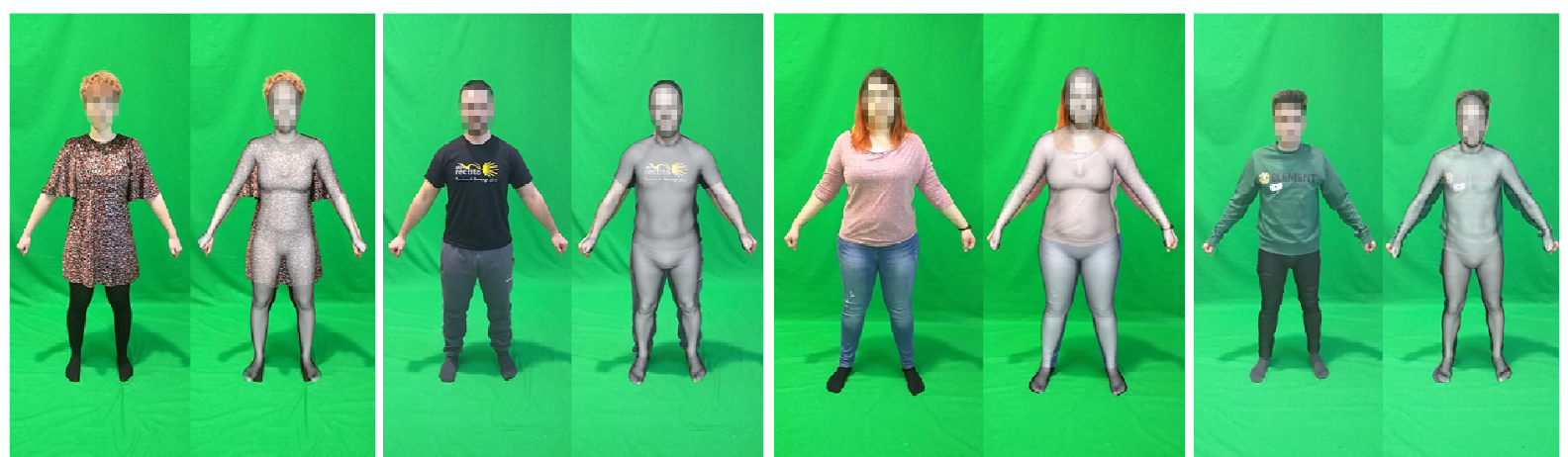

Figure 13. Initial results of ongoing research on 2D3D reconstruction people dressed with street clothes

Our 1D3D reconstruction from parameters can be easily integrated into web-based software (e.g. online stores) and can be configured to use different body measurements. These methods are, however, highly dependent on the accuracy of the inputs provided by users. Parameters such as age, height and weight can be quite reliably reported by users but the use of additional measurements is risky and, according to our testing, it did not provided significant improvements, except for bust girth. Among other factors, self-reported measuring errors are due to a poor understanding of the measuring instructions (lack of skills). In case of using additional measurements, instructions (or user training) should be carefully addressed (e.g. using video instructions [64]) and biases between the manual method and digital method should be specifically quantified and corrected. A prototype of this technology has been implemented in a cloud server accessible via API and it is ready to be tested by companies. 


\section{Acknowledgements}

This paper is supported by IVACE research programme under grant agreements IMDEEA/2018/49 (3D-BODY-HUB) and IMDEEA/2018/74 (3D-BODY-EXPERIENCE) and by European Union's Horizon 2020 research and innovation programme under grant agreements: No 779780 (BODYPASS,), and No 731885 (INKREATE, Transfer the real 3D world to interactive creative endeavours in apparel industry).

\section{References}

[1] M. Dainoff et al., "Guidelines for using anthropometric data in product design," Calif. US HFES, vol. 300, 2004.

[2] S. Pheasant, Bodyspace: Anthropometry, Ergonomics And The Design Of Work: Anthropometry, Ergonomics And The Design Of Work. CRC Press, 2014.

[3] K. M. Robinette, "Anthropometry for product design," Handb. Hum. Factors Ergon., vol. 4, pp. 330-346, 2012.

[4] NHANES, The NHANES anthropometry procedures manual. Revised 2004. 2004.

[5] NICE, Obesity: The Prevention, Identification, Assessment and Management of Overweight and Obesity in Adults and Children. London: MICE (UK), 2006.

[6] NIH, "The Practical guide: identification, evaluation, and treatment of overweight and obesity in adults.," National Institutes of Health and North American Association for the Study of Obesity and others, Publication Number 00-4084, 2000.

[7] WHO, Waist circumference and waist-hip ratio: report of a WHO expert consultation, Geneva, 8-11 December 2008. Geneva: World Health Organization, 2008.

[8] ISO 8559:1989 Garment construction and anthropometric surveys -- Body dimensions. 1989.

[9] ISO 7250-1:2017 - Basic human body measurements for technological design -- Part 1: Body measurement definitions and landmarks. 2017.

[10] ISO 18825-1:2016 - Clothing -- Digital fittings -Part 1: Vocabulary and terminology used for the virtual human body. 2016.

[11] M. Kouchi, "Anthropometric methods for apparel design: body measurement devices and techniques," in Anthropometry, Apparel Sizing and Design, Elsevier, 2014, pp. 67-94.

[12] C. C. Gordon and B. Bradtmiller, "Interobserver error in a large scale anthropometric survey," Am. J. [27] Hum. Biol., vol. 4, no. 2, pp. 253-263, 1992.

[13] C. C. Gordon et al., "1988 Anthropometric survey of US army personnel: methods and summary statistics," Anthropology Research Project Inc Yellow Springs OH, Technical Report Natick/TR-89/044, 1989.

[14] M. Kouchi et al., "Random errors in anthropometry," J. Hum. Ergol. (Tokyo), no. 25, pp. 12155-166, 1996.

[15] M. Kouchi and M. Mochimaru, "Evaluation of Accuracy in Traditional and 3D Anthropometry," 2008.

[16] J. C. Yoon and R. G. Radwin, "The accuracy of consumer-made body measurements for women's mail-order clothing," Hum. Factors J. Hum. Factors Ergon. Soc., vol. 36, no. 3, pp. 557-568, 1994.

[17] H. A. M. Daanen and F. B. Ter Haar, "3D whole body scanners revisited," Displays, vol. 34, no. 4, pp. 270-275, Oct. 2013.

[18] H. A. M. Daanen and G. J. van de Water, "Whole body scanners," Displays, vol. 19, no. 3, pp. 111120, Nov. 1998.

[19] A. Ballester et al., "3D-based resources fostering the analysis, use, and exploitation of available body anthropometric data," in 5th int. conf. on $3 D$ body scanning technologies, 2014.

[20] A. Ballester et al., "3D Body Databases of the Spanish Population and its Application to the Apparel Industry," in Proc. of 6th Int. Conf. on 3D Body Scanning Technologies, Lugano, Switzerland, 2015.

[21] D. Lacko et al., "Ergonomic design of an EEG headset using 3D anthropometry," Appl. Ergon., vol. 58, pp. 128-136, Jan. 2017.

[22] S. Verwulgen et al., "A new data structure and workflow for using 3D anthropometry in the design of wearable products," Int. J. Ind. Ergon., vol. 64, pp. 108-117, Mar. 2018.

[23] Wonsup Lee et al., "Application of massive 3D head and facial scan datasets in ergonomic head-product design," Int. J. Digit. Hum., vol. 1, no. 4, pp. 344-360, Jan. 2016.

[24] M. Kouchi and M. Mochimaru, "Errors in landmarking and the evaluation of the accuracy of traditional and 3D anthropometry," Appl. Ergon., vol. 42, no. 3, pp. 518-527, Mar. 2011.

[25] A. Kuehnapfel et al., "Reliability of 3D laser-based anthropometry and comparison with classical anthropometry," Sci. Rep., vol. 6, p. 26672, May 2016.

[26] N. Koepke et al., "Comparison of 3D laser-based photonic scans and manual anthropometric measurements of body size and shape in a validation study of 123 young Swiss men," PeerJ, vol. 5, Feb. 2017.

[27] C. Mcdonald, et al., "Working Group Progress for IEEE P3141 - Standard for 3D Body Processing," 2017, pp. 328-336.

[28] C. McDonald et al., "IEEE Industry Connections (IEEE-IC) Landmarks and Measurement Standards Comparison in 3D Body-model Processing," p. 34, 2018.

[29] M. Stahl, IEEE Industry Connections (IEEE-IC)3D Body Processing (3DBP) Initiative - An Introduction: IEEE, 2017.

[30] S. Alemany et al., "Anthropometric survey of the Spanish female population aimed at the apparel industry," in Proceedings of the 2010 International Conference on 3D Body Scanning Technologies. Lugano, Switzerland, 2010.

[31] K. M. Robinette, et al., "Civilian American and European Surface Anthropometry Resource 
(CAESAR), Final Report. Volume 1. Summary," SYTRONICS INC DAYTON OH, SYTRONICS INC DAYTON OH, Jun. 2002.

[32] R. Trieb et al., "EUROFIT—integration, homogenisation and extension of the scope of large 3D anthropometric data pools for product development," in 4th International Conference and Exhibition on 3D Body Scanning Technologies, Long Beach, CA, USA, 2013, pp. 19-20.

[33] B. Allen et al., "The Space of Human Body Shapes: Reconstruction and Parameterization from Range Scans," in ACM SIGGRAPH 2003 Papers, New York, NY, USA, 2003, pp. 587-594.

[34] A. Ballester et al., "Low-cost data-driven 3D reconstruction and its applications," in Proc. of 6th int. conf. on 3D body scanning technologies, Lugano, Switzerland, 2015.

[35] J. Boisvert, et al., "Three-dimensional human shape inference from silhouettes: reconstruction and validation," Mach. Vis. Appl., vol. 24, no. 1, pp. 145-157, Jan. 2013.

[36] H. Seo et al., "3D Body Reconstruction from Photos Based on Range Scan," in Technologies for E-Learning and Digital Entertainment, 2006, pp. 849-860.

[37] S. Zhu et al., "An efficient human model customization method based on orthogonal-view monocular photos," Comput.-Aided Des., vol. 45, no. 11, pp. 1314-1332, Nov. 2013.

[38] D. Anguelov et al., "SCAPE: shape completion and animation of people," in ACM transactions on graphics (TOG), 2005, vol. 24, pp. 408-416.

[39] J.-V. D. Dura-Gil et al., "Preliminary results of the InKreate project," Revista de Biomecánica, 2018.

[40] D. A. Hirshberg et al., "Coregistration: Simultaneous Alignment and Modeling of Articulated 3D Shape," in Computer Vision - ECCV 2012, Berlin, Heidelberg, 2012, vol. 7577, pp. 242255.

[41] M. Loper et al., "SMPL: A Skinned Multi-person Linear Model," ACM Trans Graph, vol. 34, no. 6, pp. 248:1-248:16, Oct. 2015.

[42] S. D. Walter et al., "Sample size and optimal designs for reliability studies," Stat. Med., vol. 17, no. 1, pp. 101-110, 1998.

[43] A. Ballester et al., "Data-driven three-dimensional reconstruction of human bodies using a mobile phone app," Int. J. Digit. Hum., vol. 1, no. 4, pp. 361-388, Jan. 2016.

[44] J. Long et al., "Fully convolutional networks for semantic segmentation," in 2015 IEEE Conference on Computer Vision and Pattern Recognition (CVPR), 2015, pp. 3431-3440.

[45] A. Newell et al., "Stacked Hourglass Networks for Human Pose Estimation," in Computer Vision ECCV 2016, 2016, pp. 483-499.

[46] M. Eliasziw et al., "Statistical Methodology for the Concurrent Assessment of Interrater and Intrarater Reliability: Using Goniometric Measurements as an Example," Phys. Ther., vol. 74, no. 8, pp. 777-788, Aug. 1994.

[47] B. Ng et al., "Clinical anthropometrics and body composition from 3D whole-body surface scans," Eur. J. Clin. Nutr., vol. 70, no. 11, pp. 1265-1270, Nov. 2016.

[48] J. Wang et al., "Validation of a 3-dimensional photonic scanner for the measurement of body volumes, dimensions, and percentage body fat," Am. J. Clin. Nutr., vol. 83, no. 4, pp. 809-816, Apr. 2006.

[49] T. E. Vonk and H. A. M. Daanen, "Validity and Repeatability of the Sizestream 3D Scanner and Poikos Modeling System," in 6th International Conference on 3D Body Scanning Technologies, Lugano, Switzerland, 27-28 October 2015, 2015.

[50] J. C. K. Wells et al., "Acceptability, Precision and Accuracy of 3D Photonic Scanning for Measurement of Body Shape in a Multi-Ethnic Sample of Children Aged 5-11 Years: The SLIC Study," PLoS One San Franc., vol. 10, no. 4, 2015.

[51] M. R. Pepper et al., "Validation of a 3-Dimensional Laser Body Scanner for Assessment of Waist and Hip Circumference," J. Am. Coll. Nutr., vol. 29, no. 3, pp. 179-188, Jun. 2010.

[52] Ł. Markiewicz et al., "3D anthropometric algorithms for the estimation of measurements required for specialized garment design," Expert Syst. Appl., vol. 85, pp. 366-385, Nov. 2017.

[53] J. M. Lu and M. J. J. Wang, "The Evaluation of Scan-Derived Anthropometric Measurements," IEEE Trans. Instrum. Meas., vol. 59, no. 8, pp. 2048-2054, Aug. 2010.

[54] L. D. Dekker, "3D human body modelling from range data," Doctoral, Univ. of London, 2000.

[55] K. M. Robinette and H. A. M. Daanen, "Precision of the CAESAR scan-extracted measurements," Appl. Ergon., vol. 37, no. 3, pp. 259-265, May 2006.

[56] W. C. Chumlea et al., "Replicability for anthropometry in the elderly," Hum. Biol., pp. 329337, 1984.

[57] T. G. Lohman et al., Anthropometric standardization reference manual, vol. 177. Human kinetics books Champaign, 1988.

[58] L. M. Verweij et al., "Measurement error of waist circumference: gaps in knowledge," Public Health Nutr., vol. 16, no. 02, pp. 281-288, Feb. 2013.

[59] J. Nada et al., "Intraobserver and interobserver variability of measuring waist circumference," Med. Sci. Monit., vol. 14, no. 1, pp. CR15-CR18, 2008.

[60] H. A. M. Daanen and A. Psikuta, "10 - 3D body scanning," in Automation in Garment Manufacturing, R. Nayak and R. Padhye, Eds. Woodhead Publishing, 2018, pp. 237-252.

[61] B. Bradtmiller and M. E. Gross, "3D whole body scans: Measurement extraction software validation," SAE Technical Paper, 1999.

[62] D. V. Cicchetti, "Guidelines, criteria, and rules of thumb for evaluating normed and standardized assessment instruments in psychology.," Psychol. Assess., vol. 6, no. 4, p. 284, 1994.

[63] ISO 20685:2010 - 3-D scanning methodologies for internationally compatible anthropometric databases. 2010.

[64] P. Barrios, J. Martin-Biggers, V. Quick, and C. Byrd-Bredbenner, "Reliability and criterion validity of self-measured waist, hip, and neck circumferences," BMC Med. Res. Methodol., vol. 16, no. 1, Dec. 2016. 